\title{
DETECCIÓN DE NEUROPATÍA AUTONÓMICA CARDÍACA CON MANIOBRAS DE BAJA COMPLEJIDAD EN ATENCIÓN AMBULATORIA
}

\author{
DETECTION OF CARDIAC AUTONOMIC NEUROPATHY WITH LOW \\ COMPLEXITY TESTS IN OUTPATIENT-CARE
}

\author{
Analía Yamaguchi ${ }^{1}$, Leticia Ferro ${ }^{2}$, Constanza Poma ${ }^{3}$, Raquel Urdaneta ${ }^{4}$, Graciela Fuente ${ }^{5}$
}

\section{RESUMEN}

Introducción: la neuropatía autonómica cardíaca (NAC) es una de las complicaciones más seria y menos estudiada de la diabetes. La alteración en la variabilidad de la frecuencia cardíaca es el indicador más precoz de NAC. Actualmente el diagnóstico se realiza con las maniobras de Ewing. No todos los centros del país cuentan con los profesionales capacitados y/o el equipo necesario.

Objetivo: detectar NAC con maniobras de baja complejidad y comparar resultados con las pruebas de Ewing.

Materiales y métodos: estudio observacional y transversal. Se evaluaron 24 pacientes de la Unidad de Nutrición del Hospital Carlos G. Durand entre el 1 de junio y el 30 de noviembre del 2011. Se realizaron los test de Ewing, se efectuó la medición de FC y TA en tres posiciones, y se midió el QTC.

Resultados: la media de la edad de la muestra poblacional fue de 56,54 años (47-72), y 15 eran mujeres (62,5\%). En las variables de TA y QTc, se hallaron valores dentro de lo normal. En 20 pacientes $(83,3 \%)$, los valores de FC se encontraron fuera de la normalidad, con un valor predictivo positivo del $80 \%$ (VPP) y un valor predictivo negativo del $25 \%$ (VPN), con una sensibilidad del $84 \%$ (S) y una especificidad del $20 \%$ (E). Los resultados de las pruebas de Ewing fueron patológicos en 19 pacientes (S 93\%).

Conclusión: las maniobras de baja complejidad como la falta de variedad en la frecuencia cardíaca (FVFC), no suplantan al gold estándar pero aportan un elemento de alta sospecha clínica.

Palabras clave: neuropatía autonómica cardíaca, frecuencia cardíaca, diabetes mellitus.

Revista de la Sociedad Argentina de Diabetes 2014; Vol. 48 (5-10)

1 Hospital Italiano de Buenos Aires, 2 Sanatorio Municipal Dr. Julio Méndez, ${ }^{3}$ Obra Social de los Empleados de Comercio y Actividades Civiles (OSECAC), ${ }^{4}$ y ${ }^{5}$ Hospital Carlos G. Durand. Agradecemos la colaboración de Nora Vainstein en este trabajo E-mail: prana.samadhi@gmail.com

Correspondencia: Av. Nazca 3177, piso 30 depto. 39 (C1417CVB), CABA, Argentina

\section{ABSTRACT}

Introduction: the cardiac autonomic neuropathy (CAN) is one of the most serious and less studied diseases associated with diabetes. The lack of variability in the cardiac rate is the most premature indicator for CAN. At present, the diagnosis is made with Ewing tests. In Argentina, there are just few medical centers and professionals with the expected experience and necessary equipment.

Aim: to evaluate the sensitiveness of low complexity tests for CAN premature detection, comparing those results with the ones of Ewing tests.

Methodology and materials: observational and transversal study. Between June 1st and November 30th 2011, 24 patients were included in the model of the study made in the "Unidad de Nutrición del Hospital C. Durand." Ewing tests were made and heart rate and blood pressure in three positions, also QT were measured. Results: the age mean of the sample was 56,54-year old patients (47to72) and 15 were women (62,5\%). Blood pressure and QTC values were found between normal parameters. However, for $83,3 \%$ of the patients heart rate values were found outside normal parameters, with a positive predictive value of $80 \%$ (PPV) and a negative predictive value of 25\% (NPV), with a sensitiveness of $84 \%$ (S) and specificity value of $20 \%$ (E). The results of Ewing tests were pathologic for $93 \%$ of the patients (S 19).

Conclusion: low complexity tests as well as lack of variability of heart rate do not replace gold standard but contribute as evidence to have some doubts on common clinical practices.

Key words: cardiac autonomic neuropathy, heart rate, diabetes mellitus.

Revista de la Sociedad Argentina de Diabetes 2014; Vol. 48 (5-10)

\section{INTRODUCCIÓN}

La neuropatía autonómica cardíaca (NAC) es una de las complicaciones más seria y menos estudiada de la diabetes mellitus (DM). Los informes de prevalencia varían ampliamente de acuerdo con los métodos de estudio empleados, los cuales oscilan entre el 10 y el $90 \%$ de los diabéticos examinados, afectando a un tercio de los que padecen DM2'.

La NAC representa una de las causas más im- 
portantes de morbilidad y mortalidad en pacientes diabéticos y se asocia a un riesgo elevado de arritmias cardíacas y muerte súbita².

Informes de los principales ensayos clínicos establecen la importancia del control glucémico en relación al riesgo metabólico ya evidenciado en el estudio de Framingham ${ }^{3,4}$, al igual que otros estudios que demostraron que la DM es un factor de riesgo para las enfermedades cardiovasculares. Del mismo modo, la DM aumenta la morbilidad y mortalidad, siendo necesario considerar a los factores cardiovasculares como la primera causa de mortalidad en la DM2. La NAC es, por lo tanto, una de las formas más comunes de disfunción autonómica en la DM y una de las anormalidades asociadas con el control del ritmo cardíaco y la dinámica vascular ${ }^{5,6}$.

El insatisfactorio estado de conocimiento de esta afección se debe a los diferentes criterios empleados en la selección de las muestras, el relativo y escaso número de pacientes estudiado en diferentes regiones del mundo que no permite una visión más global del grado de afectación de esta complicación y, a su vez, la falta de consenso en relación a la utilización de la batería estandarizada de pruebas autonómicas cardíacas.

Frecuentemente la NAC es asintomática; sus manifestaciones clínicas se caracterizan por: anormalidades en el control de la variación de la frecuencia cardíaca, así como de alteraciones en el control de la tensión arterial ante exigencias fisiológicas. No obstante, el compromiso de la variabilidad de la frecuencia cardíaca es el indicador más precoz de NAC. Su presencia puede limitar la capacidad de ejercicio del individuo e incrementar el riesgo de un evento cardiovascular durante la práctica ${ }^{7,8}$.

El diagnóstico de NAC se realiza con las pruebas de Ewing que requieren de personal capacitado y un software adecuado. Es importante destacar que prácticamente ninguno de los centros de salud del país posee estos materiales por su elevado costo. Creemos importante que los médicos de atención primaria cuenten con una herramienta de baja complejidad para la evaluación y detección en forma temprana de la NAC, con el fin de establecer los ajustes necesarios para la optimización del tratamiento de la enfermedad de base y de la complicación propiamente dicha.

Por tal motivo, el objetivo de este trabajo es comparar las maniobras de baja complejidad con las pruebas de Ewing para determirnar cuál de ellas presenta mayor sensibilidad para detectar NAC asintomática en pacientes con DM2. El objetivo secundario es observar las diferencias que presentan los pacientes según tengan menos o más de cinco años desde el diagnóstico de DM2.

\section{Objetivo}

Diagnosticar NAC con maniobras de baja complejidad (FC, tensión arterial, ECG con medición del Qt), evaluar la posible utilidad clínica de estos métodos y comparar la falta de variabilidad de la FC con las pruebas de Ewing.

\section{MATERIALES Y MÉTODOS}

- Tipo de investigación: estudio observacional, transversal y comparativo para determinar la sensibilidad de las maniobras de baja complejidad, comparadas con las pruebas de Ewing.

- Población: se incluyeron pacientes de ambos sexos, de entre 40 y 80 años de edad, con diagnóstico de DM2 que concurrieron los jueves, entre las 8 y 12 horas, desde el $1^{\circ}$ de junio hasta el 30 de noviembre de 2011, a la Unidad de Nutrición del Hospital Carlos G. Durand. En dicha Unidad, en forma sistemática, se realizó la detección de hipotensión ortostática en la consulta ambulatoria y se solicitó ECG; además se contaba con la infraestructura y el software necesarios para realizar las pruebas de Ewing. El número de pacientes evaluados fue de 243; cumplieron criterios de inclusión 29, y cinco pacientes no se presentaron para realizar las pruebas autonómicas, por lo cual la muestra poblacional resultó en 24 pacientes estudiados.

\section{Criterios de inclusión}

- Pacientes de ambos sexos con diagnóstico de DM2 entre 40 y 80 años 9 .

- Pacientes con factores de riesgo cardiovascular (HTA, tabaquismo, dislipemia) $)^{10}$.

- Pacientes con retinopatía o nefropatía ${ }^{11}$.

\section{Criterios de exclusión}

- Antecedentes de isquemia coronaria, insuficiencia cardíaca, arritmias.

- Taquicardia en reposo.

- Pacientes con neuropatía periférica de causa diabética y no diabética.

- Bajo tratamiento con drogas que prolongan el QT (antiarrítmicos, antihistamínicos, antipsicóticos, macrólidos, imidazólicos, cocaína). 
- Bajo tratamiento con betabloqueantes, bloqueantes cálcicos, bloqueantes alfa, digitálicos, amiodarona u otros fármacos con efecto cronotrópico negativo.

- Bajo tratamiento con vasodilatadores (por taquicardia refleja).

- Hipotiroidismo o hipertiroidismo.

- Pacientes con serología positiva para Chagas asociada a alteraciones electrocardiográficas.

- Pacientes con glucemia menor a $70 \mathrm{mg}$ en el momento de la evaluación.

- Pacientes embarazadas.

A quienes concurrieron al consultorio y cumplieron con los criterios de inclusión, se les efectuaron las maniobras de Ewing 9 .

Los test de Ewing comprenden cinco pruebas a las cuales, por razones didácticas, se las suele dividir en simpáticas y parasimpáticas. Se aconseja realizarlas luego que el paciente permanezca varios minutos en reposo, sin haber ingerido medicaciones que modifiquen la FC, sin fumar, ni presentar hipoglucemia, ni fiebre. El ambiente donde se concreten debe ser de temperatura agradable.

De los cinco test de Ewing para pacientes ambulatorios, sólo tres son actualmente recomendados por la Asociación Americana de Diabetes (ADA). Los tres test parasimpáticos se realizaron con el paciente conectado al electrocardiógrafo y a la computadora, con el software adecuado para la medición de los complejos RR correspondientes (AKONIC PC).

Actualmente la Academia Americana de Neurología (ANA) recomienda en el momento del diagnóstico de DM2 realizar las siguientes maniobras: test de respiración profunda, maniobra de Valsalva y prueba ortostática. Estas pruebas deben repetirse anualmente. Las mismas tienen una buena reproducibilidad y especificidad superior al $91 \%$, y una sensibilidad del 93\% (prueba de respiración profunda y prueba ortostática) al 98\% (Valsalva).

La medición del segmento QT también es de utilidad para evaluar la función autonómica cardía$c a$, ya que existe evidencia de la relación entre la muerte súbita y la longitud de este segmento que es considerado indicador de la repolarización cardíaca. Su regulación depende del sistema nervioso simpático, y el valor normal del QT es $=0<a$ 440 milisegundos $9,12,13,14$.

En nuestro trabajo un operador realizó los test de Ewing y un investigador, que desconocía los resultados, efectuó en los pacientes las siguientes maniobras:

- Frecuencia cardíaca central: con el paciente en decúbito dorsal, sentado y de pie (en ese orden), se definió su variación como el aumento de 20 latidos por minuto de la frecuencia cardíaca entre cada medición. Consideramos el resultado de la prueba patológica cuando faltó la variación; es decir, que el aumento fue menor o igual a 10 latidos por minuto.

- Tensión arterial: medida con el paciente en reposo (por lo menos 30 minutos), en una habitación tranquila antes de realizar las mediciones, con el brazo a la altura cardíaca (sea cual fuere la posición del individuo), libre de toda compresión, pronado y con el antebrazo apoyado sobre una superficie firme (JNC7). Se realizaron mediciones en decúbito dorsal, sentado y de pie (en ese orden). Se definió como prueba patológica a la caída de $20 \mathrm{mmHg}$ y $10 \mathrm{mmHg}$ en la presión sistólica y diastólica respectivamente ${ }^{12,13,14}$.

- ECG de 12 derivaciones: se realizó medición del intervalo QT en las derivaciones DII, V2 ó V3 por ser las derivaciones en las se registra mayor duración del mismo. Se efectuó corrección de los valores de QT según fórmula QTc = QT /(sqrt RR) y finalmente se calculó el promedio con el fin de evaluar la prolongación de dicho intervalo, considerando como normal una duración de hasta 440 milisegundos ${ }^{9}$.

En todos los pacientes, incluso, se evaluaron peso, talla, IMC y se registraron los siguientes datos de la historia clínica: sexo, edad, años desde el diagnóstico de DM2, presencia o ausencia de dislipemia, HTA, retinopatía y nefropatía, además del tratamiento antidiabético (agentes orales y/o insulina).

Se solicitaron análisis bioquímicos y serología en el laboratorio del Hospital Carlos G. Durand, al inicio del estudio: glucemia, \% de HbA1c (método turbidimetría), TSH y serología para Chagas.

- Análisis estadístico: las variables cualitativas se expresan en porcentajes o proporciones. Se aplicó test chi cuadrado con corrección de continuidad para establecer posibles asociaciones. Los valores con distribución normal se informaron como media con desvío estándar, y el rango de valores con distribución no normal se informó como mediana con el desvío en los percentilos 25 y 75 . Se consideró estadísticamente significativa una p menor a 0,05. 
Se realizó un análisis de grado de concordancia a través del índice kappa de Cohen en las variables de patología autonómica y frecuencia cardíaca.

\section{RESULTADOS}

Del total de la muestra, $62,5 \%$ era mujeres (15). La media de la edad de la muestra poblacional fue de 56,54 años.

Al analizar el IMC se evidenció que del total de la muestra, la mayoría de los pacientes presentaba sobrepeso (37,5\%) y obesidad (33,3\%). Según las metas de la Asociación Americana de Diabetes $\left(\mathrm{ADA}^{15}\right)$ para glucemia y HbA1c, se observó que se encontraban dentro de los objetivos el 54 y el $79 \%$ de los pacientes respectivamente. Con respecto a las comorbilidades y complicaciones de la DM2, se hayó que presentaban HTA el 66,6\% $(n=16)$, dislipemia el $66,6 \%(n=16)$, retinopatía el $25 \%(n=6)$ y nefropatía el 20,8\% ( $n=5)$ (Tabla 1$)$.

Según años de diagnóstico de DM2, se observó que presentaban menos de cinco años el $58,4 \%(n=14)$ de la muestra, y el 41,6\% ( $n=10)$ con más de cinco años.

Al analizar los resultados de las variables de TA y QTc, en toda la muestra se hallaron valores dentro de los parámetros de la normalidad. Sin embargo al analizar los valores de la FC, se observó que en 20 pacientes $(83,3 \%)$, los valores se encontraron fuera de los puntos de corte que definen la normalidad, con un valor predictivo positivo (VPP) del $80 \%$ y un valor predictivo negativo (VPN) del $25 \%$, con una sensibilidad del $84 \%$ (S) y una especificidad del $20 \%$ (E). Los resultados de las pruebas de Ewing fueron patológicos en 19 pacientes, teniendo en cuenta que dichas pruebas poseen una especificidad superior al $91 \%$ y una sensibilidad del 93\% (prueba de la respiración profunda y prueba ortostática) al 98\% (Valsalva) (Gráfico 1).

Dentro del subgrupo con menos de cinco años del diagnóstico $(n=14)$ encontramos que los test de Ewing resultaron patológicos en nueve de ellos. Dentro de los pacientes con pruebas de Ewing patológicas, dos presentaron FC normal, y dentro de aquellos con pruebas de Ewing normales $(n=5)$, un paciente presentó $F C$ normal, con una sensibilidad del $78 \%$ y una especificidad del $20 \%$ para la variable FC.

En aquellos pacientes con más de cinco años desde el diagnóstico de DM ( $n=10)$, se hallaron en todos los casos pruebas de Ewing patológicas; de éstos, sólo un paciente presentó FC normal, con una sensibilidad del 90\% (Gráfico 2).

Analizando el subgrupo de pacientes con menos de cinco años desde el diagnóstico de DM2, se observó que el $57,1 \%$ de la muestra era de sexo femenino y la mediana de edad de 55 años. En este subgrupo, el 78,6\% presentó HTA y el $57,1 \%$ dislipemia, mientras que nefropatía y retinopatía estuvieron presentes en el $21,4 \%$ de los pacientes (Gráfico 2).

Cuando se estudiaron los resultados y correlacionando la glucemia en ayunas y la $\mathrm{HbA} 1 \mathrm{c}$, las medias fueron de $108 \mathrm{mg} / \mathrm{dl}$ y 5,91\% respectivamente. En este subgrupo el $72 \%$ de los pacientes estaba en meta para glucemia en ayunas y el $100 \%$ en meta para $\mathrm{HbA} 1 \mathrm{c}$ (Tabla 2).

Respecto al subgrupo de pacientes con más de cinco años desde el diagnóstico de DM2, se observó que el $70 \%$ era de sexo femenino y la mediana de edad de 58,9. Se observó HTA en un $50 \%(n=5)$, dislipemia en un $80 \%(n=8)$, retinopatía en un 30\% ( $n=3)$ y nefropatía en un $20 \%(n=2)$ (Gráfico 2).

En estos pacientes se evidenció que el 10\% estaba en meta para glucemia en ayunas, con una media de $170,10 \mathrm{mg} / \mathrm{dl}$ y el $50 \%$ para $\mathrm{HbA} 1 \mathrm{c}$, con una media de 7,56\% (Tabla 2).

Existieron diferencias significativas entre ambos subgrupos con respecto a la media de $\mathrm{HbA} 1 \mathrm{c}$ y de las glucemias en ayunas. Dichas medias fueron significativamente mayores en los pacientes con más de cinco años de diagnóstico de DM ( $p<0,0001$ para ambas variables) (Tabla 2).

Al examinar las comorbilidades, se observó mayor porcentaje de HTA y menor porcentaje de dislipemia en el subgrupo de menos de cinco años de diagnóstico; en referencia a las complicaciones de la DM, presentaron menor retinopatía y mayor nefropatía los pacientes de este mismo subgrupo (Tabla 1, Gráfico 2). El grado de acuerdo del índice de kappa de Cohen entre las variables de pruebas de Ewing y la FC fue muy pobre ( $p 0,20)$.

\section{DISCUSIÓN}

De las maniobras de baja complejidad (TA, QTc, FC) efectuadas a los pacientes en este trabajo, la única que tuvo modificaciones fue la FC, más específicamente la falta de variabilidad de la FC, teniendo una mayor sensibilidad para los pacientes con más de cinco años de diagnóstico de DM2. Por otra parte, el grado de concordancia por el índice de kappa de Cohen fue bajo. 
Por lo tanto, utilizar la FC en el consultorio de atención ambulatoria presenta una buena sensibilidad para realizar la detección, pero no suplanta al gold estándar (test de Ewing).

Al subdividir en dos grupos (menos y más de cinco años de diagnóstico de DM2) a la muestra estudiada, se observó que los pacientes con más de cinco años de diagnóstico de DM2 presentaron en su totalidad resultados patológicos de la evaluación autonómica. Esto pone de manifiesto que a mayor tiempo de diagnóstico de DM2, mayor riesgo de complicaciones.

Al comparar ambos subgrupos, se hallaron diferencias significativas en el control metabólico, principalmente en relación a la HbA1c $(p<0,001)$ y a las glucemias en ayunas $(p<0,001)$, destacando que a más años de diagnóstico de DM2, mayor deterioro metabólico y, por lo tanto, mayor riesgo de complicaciones.

Al analizar los resultados se determinó que, en el subgrupo de más de cinco años desde el diagnóstico de DM2, el porcentaje de pacientes con HTA fue menor que en el subgrupo de menos de cinco años (Gráfico 2). A partir de esta observación, surge como interrogante si no nos encontraríamos frente a pacientes con HTA oculta.

\begin{tabular}{|l|c|c|c|}
\hline $\begin{array}{c}\text { Comorbilidades } \\
\text { y complica- } \\
\text { ciones }\end{array}$ & Total (\%) & $<\mathbf{5}$ años (\%) & $>\mathbf{5}$ años (\%) \\
\hline HTA & 66,6 & 78,6 & 50 \\
\hline Dislipemia & 66,6 & 57,1 & 80 \\
\hline Nefropatía & 20,8 & 21,4 & 20 \\
\hline Retinopatía & 25 & 21,4 & 30 \\
\hline
\end{tabular}

Tabla 1: Comorbilidades y complicaciones.

\begin{tabular}{|l|r|r|r|r|l|}
\hline & N & Media & $\begin{array}{r}\text { IC 95\% } \\
\text { Inferior }\end{array}$ & $\begin{array}{l}\text { IC 95\% } \\
\text { Superior }\end{array}$ & $\begin{array}{l}\text { Des- } \\
\text { viación } \\
\text { estándar }\end{array}$ \\
\hline$<5$ años & & & & & \\
\hline HbA1c & 14 & 5,914 & 5,683 & 6,145 & 0,3997 \\
\hline Glucemia & 14 & 108,50 & 90,09 & 126,91 & 31,881 \\
\hline$>5$ años & & & & & \\
\hline HbA1c & 10 & 7,560 & 6,024 & 9,096 & 21,475 \\
\hline Glucemia & 10 & 170,10 & 125,34 & 214,86 & 62,573 \\
\hline
\end{tabular}

Tabla 2: Glucemia y HbA1c.

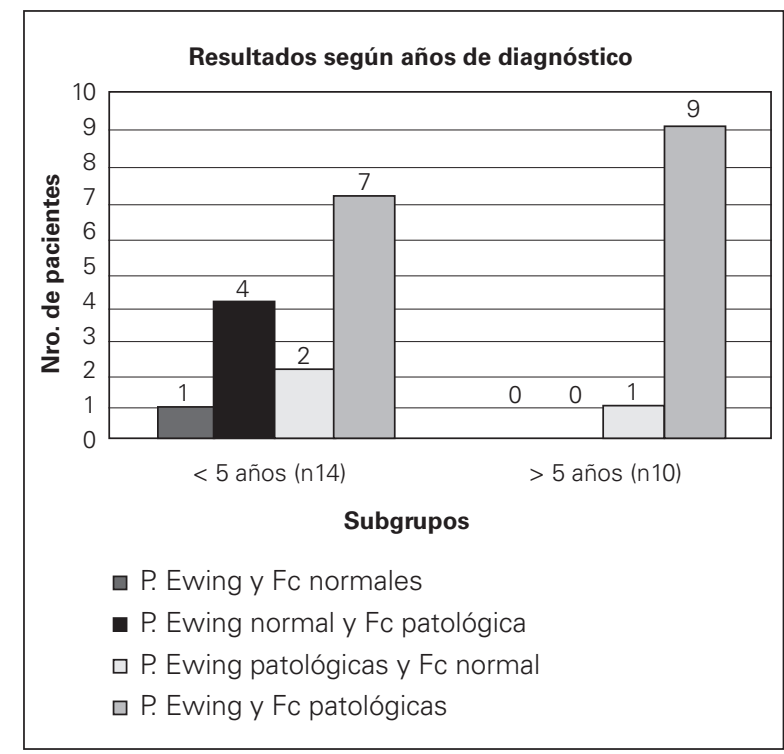

Gráfico 1: Resultados de FC y maniobras de Ewing por subgrupos.

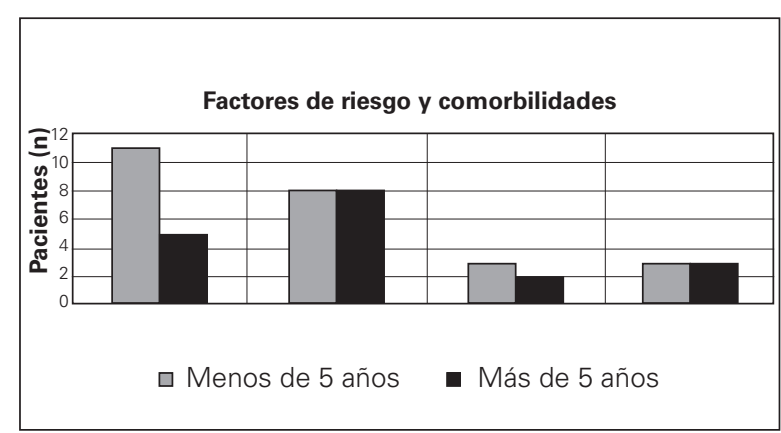

Gráfico 2: Comorbilidades y complicaciones en ambos grupos.

\section{CONCLUSIÓN}

La falta de variabilidad de la FC no puede utilizarse como herramienta diagnóstica pero demuestra ser una variable a tener en cuenta como screening en ausencia de disponibilidad de las pruebas de Ewing. La falta de variabilidad de la FC debería hacer sospechar sobre la presencia de NAC asintomática en pacientes con DM2. Debido a los datos recogidos en la población analizada en el presente trabajo, podemos afirmar que a todos los pacientes a quienes se les detecte falta de variabilidad en la FC, debería realizárseles las mencionadas pruebas para eventualmente diagnosticar NAC e implementar el tratamiento precoz y oportuno.

Si bien nuestro trabajo no aporta elementos nuevos para el diagnóstico de NAC, intentamos 
mejorar la detección temprana. Reconocemos como limitación el pequeño tamaño de la muestra estudiada que podría influir en los resultados esta- dísticos, por lo cual consideramos que se requieren estudios con mayor cantidad de pacientes con el fin de ratificar o no dichos resultados.

\section{REFERENCIAS}

1. Vicente Herrero MT, Ramírez Iñiguez de la Torre MA, Capdevila García LM, Terradillos García MJ, López González AA. Complicaciones neurológicas en la diabetes. Grupo de trabajo de la AEEMT, 2010.

2. Fearley RD, Low PA, Thomas JE. Thermoregulatory sweating abnormalities in diabetes mellitus. Mayo Clin. Proc. 1989; 64:617-28.

3. Kannel WB, Hjortland M, Castelli WP. Role of diabetes in congestive heart failure: the Framingham Study. Am. J Cardiol. 1974; 34:29-34.

4. Página web: www.framinghamheartstudy.org/about/spanish.html.

5. Rutter MK, Parise H, Benjamin EJ, Levy D, Larson MG, Meigs JB, Nesto RW, Wilson PW, Vasan RS. Impact of glucose intolerance and insulin resistance on cardiac structure and function: sex related differences in the Framingham Heart Study. Circulation 2003; 107:448-454.

6. Feuvray D, Darmellah A: Diabetes-related metabolic perturbations in cardiac myocyte. Diabetes Metab. 2008; 34 (Suppl 1): S3-S9.

7. Poanta L, Porojan M, Dumitrascu DL. Real Heart rate variability and diastolic dysfunction in patients with type 2 diabetes meIlitus. Acta Diabetol. 2011 Sep; 48(3):191-6. Epub 2011, Feb 5.

8. Cardiac autonomic neuropathy in diabetes. From the Department of Internal Medicine, Division of Metabolism, Endocrinology and Diabetes, University of Michigan, Ann Arbor, Michigan. Diabetes Care, Vol. 33, N², February 2010.

9. Actualización de Consenso de Neuropatía Diabética. Bahía Blanca, 29 de mayo de 2009. Comité de Neuropatía Diabética de la Sociedad Argentina de Diabetes.

10. Vinik A, Erbas T. Diabetic autonomic neuropathy, 2004. En:The diabetes mellitus manual. New York: McGraw Hill. 2004:351.

11. Writing team for the diabetes control and complications trial/ epidemiology of diabetes interventions and complications research group. Sustained effect of intensive treatment of type 1 diabetes mellitus on development and progression of diabetic nephropathy: the epidemiology of diabetes interventions and complications (EDIC) study. JAMA 2003; 290: 2159-2167.

12. Spallone V, Morganti R, Fedele T, D'Amato C, Maiello MR. Reappraisal of the diagnostic role of orthostatic hypotension in diabetes. Clin. Auton. Res. 2009;19: 58-64.

13. Consensus Committee of the American Autonomic Society and the American Academy of Neurology. Consensus statement on the definition of orthostatic hypotension, pure autonomic failure, and multiple system atrophy. Neurology 1996; 46:1470.

14. Lykke JA, Tarnow L, Parving HH, Hilsted J. A combined abnormality in heart rate variation and QT corrected interval is a strong predictor of cardiovascular death in type 1 diabetes. Scand J Clin Lab Invest 2008; 12:1-6.

15. American Diabetes Association. Diabetes Care 2008; 31(Suppl 1): S12-S54. 